\title{
Biologic Response of Inguinal Hernia Prosthetics: A Comparative Study of Conventional Static Meshes Versus 3D Dynamic Implants
}

\author{
*Giuseppe Amato, *Giorgio Romano, *Antonino Agrusa, $†$ Salvatore Marasa, \\ *Gianfranco Cocorullo, *Gaspare Gulotta, ¥Thorsten Goetze, and §Roberto Puleio
}

*Departments of General Surgery and Emergency and Organ Transplantation and †Pathologic Anatomy and Histology, University of Palermo; \$Department of Pathologic Anatomy and Histology, Istituto Zooprofilattico Sperimentale della Sicilia, Palermo, Italy; and $¥$ Department of General and Visceral Surgery, Ketteler Krankenhaus, Offenbach, Germany

\begin{abstract}
Despite improvements in prosthetics and surgical techniques, the rate of complications following inguinal hernia repair remains high. Among these, discomfort and chronic pain have become a source of increasing concern among surgeons. Poor quality of tissue ingrowth, such as thin scar plates or shrinking scars - typical results with conventional static implants and plugs-may contribute to these adverse events. Recently, a new type of 3D dynamically responsive implant was introduced to the market. This device, designed to be placed fixation-free, seems to induce ingrowth of viable and structured tissue instead of regressive fibrotic scarring. To elucidate the differences in biologic response between the conventional static meshes and this 3D dynamically responsive implant, a histological comparison was planned. The aim of this study was to determine the quality of tissue incorporation in both types of implants excised after short, medium, and long periods
\end{abstract}

post-implantation. The results showed large differences in the biologic responses between the two implant types. Histologically, the 3D dynamic implant showed development of tissue elements more similar to natural abdominal wall structures, such as the ingrowth of loose and well-hydrated connective tissue, well-formed vascular structures, elastic fibers, and mature nerves, with negligible or absent inflammatory response. All these characteristics were completely absent in the conventional static implants, where a persistent inflammatory reaction was associated with thin, hardened, and shrunken fibrotic scar formation. Consequently, as herniation is a degenerative process, the $3 \mathrm{D}$ dynamic implants, which induce regeneration of the typical groin components, seem to address its pathogenesis. Key Words: Inguinal hernia-Herniorrhaphy-Biomaterials -Prostheses-Implants-Tissue regeneration-Tissue scaffolds.
Inguinal hernia repair is one of the most commonly performed surgical procedures. In the USA alone, over 800000 hernia repairs are carried out yearly (1). More than 2 million prosthetic implants are used yearly in patients undergoing inguinal hernia repairs (2). Nevertheless, the rate of intra- and postoperative complications remains unacceptably high. Despite the continual progress of surgical techniques and materials, bleeding, hematoma, postoperative

doi:10.1111/aor.12416

Received May 2014; revised August 2014.

Address correspondence and reprint requests to Prof. Giuseppe Amato, Department of General Surgery and Emergency, University of Palermo, Via Del Vespro 129, Palermo, Sicily 90127, Italy. E-mail: amatomed@gmail.com discomfort/pain, and recurrence still remain unresolved problems. These complications often lead to reductions in patient quality of life and have a negative impact on healthcare economics. Growing concerns have been voiced in recent literature regarding poor outcomes following inguinal hernia repair. Deep fixation is acknowledged as one cause of many of the common complications, including chronic pain (3-6). For this reason, implant fixation has become a source of continuous discussion among herniologists. Currently, the concept of surgical therapy is based on the principle of groin strengthening by inducing the ingrowth of a regressive, inelastic fibrotic scar plate, which describes the biologic response of conventional flat meshes. As the inguinal region is one of the most motile areas of the body, it is surprising that all 
prosthetics used for hernia repair are static devices. Recent evidence indicates that inguinal hernia is a degenerative disease (7-11). Therefore, to repair abdominal wall defects, the goal of the employed therapy should be to regenerate typical abdominal wall tissue components. Moreover, to better mimic the physiology and biodynamics of the groin, hernia repair should be carried out using dynamically compliant devices.

To satisfy these requirements, a new prosthesis was recently developed that is made from the same material as conventional implants (polypropylene), but is structured to respond to the kinetic stresses of the inguinal region due to its intrinsic dynamic compliance. This prosthesis has a 3D multilamellar structure and can be implanted fixation-free into the hernia opening because of its inherent centrifugal expansion. As a result, the biologic response to this implant is very different from the response to static meshes. In fact, based on experimental tests on porcine models, tissue incorporation within the 3D structure displays all distinctive elements of the abdominal wall: loose and well-hydrated connective tissue, elastic fibers, mature vascular structures, and nerves (12). The ingrowth of these tissue components shows a steady progression over time. We are confident that this difference in biological response is due to the dynamic compliance of the implant. This kind of tissue ingrowth seems to be extremely helpful in eliminating postoperative discomfort and pain in inguinal hernia repair patients. To better elucidate the differences in biologic response between conventional static and 3D dynamic implants, we performed a comparative study on tissue incorporation in implants excised from patients at short, medium, and long intervals after surgery.

\section{PATIENTS AND METHODS}

The study was approved by the ethics committee of the Landesärztekammer Hessen. The model of histological comparison was chosen to highlight tissue incorporation in conventional flat meshes for inguinal hernia repair and 3D dynamic implants after 3-5 weeks (short-term), 3-4 months (mid-term), and 6-8 months (long-term) after implantation in the inguinal region. We examined 15 biopsies removed during interventions for recurrent inguinal hernia from conventional prostheses of different brands. All conventional prostheses were lightweight, large-pore polypropylene, implanted during Lichtenstein hernia repair (Fig. 1A). The removed flat meshes were produced by Angiologica (S. Martino Siccomario, Pavia, Italy; Repol Angimesh, 3 units), Assut Europe (Magliano de Marsi, Italy; Assumesh, 5 units), JSC (Vilnius, Lithuania; Radomesh, 3 units), and B. Braun (Melsungen, Germany; Premilene, 4 units). Three biopsies were taken from prostheses during postoperative weeks 3 to 5 , five biopsies were performed during postoperative months 3 to 4 , and the remaining seven flat mesh biopsies were taken 6 to 8 months after placement. Fifteen 3D dynamic implants were examined, all of which were also made from low-weight, large-pore ProFlor-type polypropylene (manufactured under license by Insightra Medical Inc., Irvine, CA, USA) (Fig. 1B). The biopsies were performed in patients undergoing surgery in the inguinal region for various pathologies (e.g., concomitant hydrocele ipsilateral to the previous hernia, overlooked multiple hernia protrusions). Of the 15 , four patients were biopsied between 3 and 5 weeks postoperatively, five patients were biopsied between 3 and 4 months postoperatively, and the last

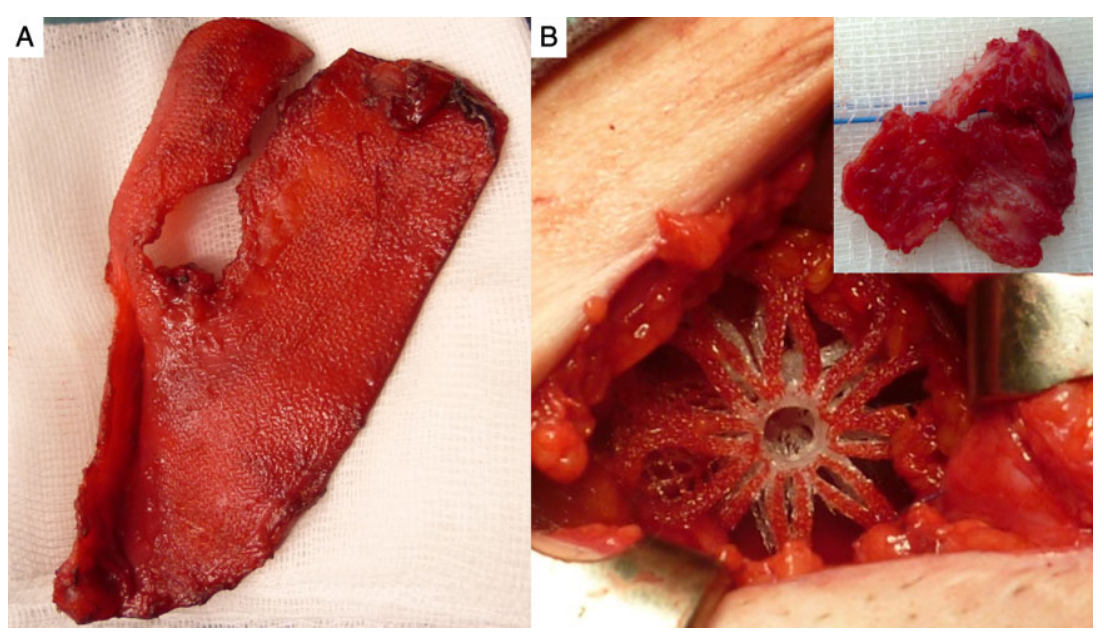

FIG. 1. (A) Conventional flat mesh excised during recurrent inguinal hernia procedure 8 month after Lichtenstein repair. (B) 3D dynamic implant placed fixation free within the internal ring during indirect inguinal hernia repair. The inset shows a biopsy specimen of the $3 \mathrm{D}$ dynamic implant excised 8 months after implantation. 
six patients were biopsied between 6 and 8 months postoperatively.

\section{Histology and immunohistochemistry}

Tissues removed from both types of implants were fixed in $10 \%$ phosphate-buffered formalin and then embedded in paraffin. Sections $(4 \mu \mathrm{m}$ thick $)$ were cut and stored at room temperature until use. Routine histology (hematoxylin-eosin staining, $\mathrm{H} \& \mathrm{E}$ ) was performed in order to evaluate the basic histomorphological features of the tissues. The avidin-biotin-peroxidase complex method (LSAB, Dako, Santa Clara, CA, USA) was used for immunohistochemical studies. Tissue sections were sequentially dewaxed through a series of xylene, graded alcohol, and water immersion steps. Antigen was retrieved in citrate buffer ( $\mathrm{pH}$ 6.0) with microwave digestion ( 2 cycles of $750 \mathrm{~W}$ for 5 min each). Endogenous peroxide activity was suppressed by incubating the sections with $0.3 \%$ hydrogen peroxidase in methanol for $30 \mathrm{~min}$ at room temperature. After three rinses for $10 \mathrm{~min}$ each in phosphate buffered saline (PBS), all tissue sections were incubated with $1 \%$ bovine serum albumin for $30 \mathrm{~min}$ at room temperature. A polyclonal sheep antibody against human enolase 2 (neuron-specific enolase; R\&D Systems, Minneapolis, MN, USA) was used as a primary antibody. Primary antibody was diluted in PBS with $0.1 \%$ normal albumin serum (1:100), and sections were further incubated overnight at $4^{\circ} \mathrm{C}$. After incubation, the slides were rinsed three times with PBS for $5 \mathrm{~min}$, and a biotinylated secondary immunoglobulin (LSAB, Dako) was applied for $30 \mathrm{~min}$ at room temperature. After two rinses with PBS for $5 \mathrm{~min}$ each, tissue sections were incubated in PBS for $1 \mathrm{~h}$ at room temperature with streptavidinhorseradish peroxidase conjugate. All tissue sections were rinsed three times with Tris-buffered saline (TBS), incubated with the chromogen 3-3'diaminobenzidine tetrahydrochloride (Dako) diluted to $0.035 \%$ in TBS for $1 \mathrm{~min}$, rinsed in tap water, and counterstained with Mayer's hematoxylin. The specific primary antibody was replaced with PBS in tissue sections used as negative controls.

\section{Histochemistry methods}

Sections were also analyzed by Weigert's and azan-Mallory staining. Weigert's method was performed to determine the presence and amount of elastic fibers within the ingrown tissue by enabling the visualization of elastic system fibers. The method is based on the affinity of elastic fibers towards resorcin-fuchsin, a precipitate resulting from a reaction among resorcin, basic fuchsine, and ferric chloride. For the azan-Mallory method, two acid dyes were used: azocarmine and aniline blue. Azocarmine, a cytological stain, is combined with an aniline blue counterstain after mordanting with phosphotungstic acid. Azan-Mallory stains collagen blue, muscle a reddish color, and chromatin and erythrocytes red.

\section{Digital image analysis}

Images of stained slides were captured using a Leica DMR microscope equipped with a Leica DFC 320 digital camera (Leica Microsystems, Wetzlar, Germany).

\section{Assessment of histopathological parameters}

The histologic sections were evaluated by a medical (SM) and a veterinary (RP) pathologist. Samples were examined by light microscopy at high power, and the mesh-tissue interface was observed to perform quantitative histological analysis to evaluate cellular infiltration with particular cell types, connective tissue, fibrosis, and neovascularization. Ten non-overlapping fields at $20 \times$ magnification were observed for each histological sample. The assessment parameters are highlighted in Table 1.

\section{Inflammatory infiltration}

The inflammatory response was determined using a scoring system based on the presence of neutrophils, lymphocytes, macrophages, and plasma cells. At 20× magnification, the cellular infiltration count was based on 10 sites at the host-mesh interface (Fig. 2). The presence of neutrophils in the tissue equates to acute inflammation, while the presence of lymphocytes, macrophages, and plasma cells is an indicator of chronic inflammation.

\section{Neovascularization}

Blood vessels were counted in 10 high-power fields (HPFs, 200×), with a score of 0 indicating no more than one blood vessel in the mesh, and a score of 3 indicating that new blood vessels were evident within the implant interstices, forming an interconnected microvascular network (Fig. 2).

\section{Connective tissue ingrowth}

Determining the content of connective elements is an important diagnostic and prognostic test. Connective tissue components were highlighted by azanMallory and H\&E staining. The score was based on the number of layers at the mesh-tissue interface. Connective ingrowth was categorized based on the 

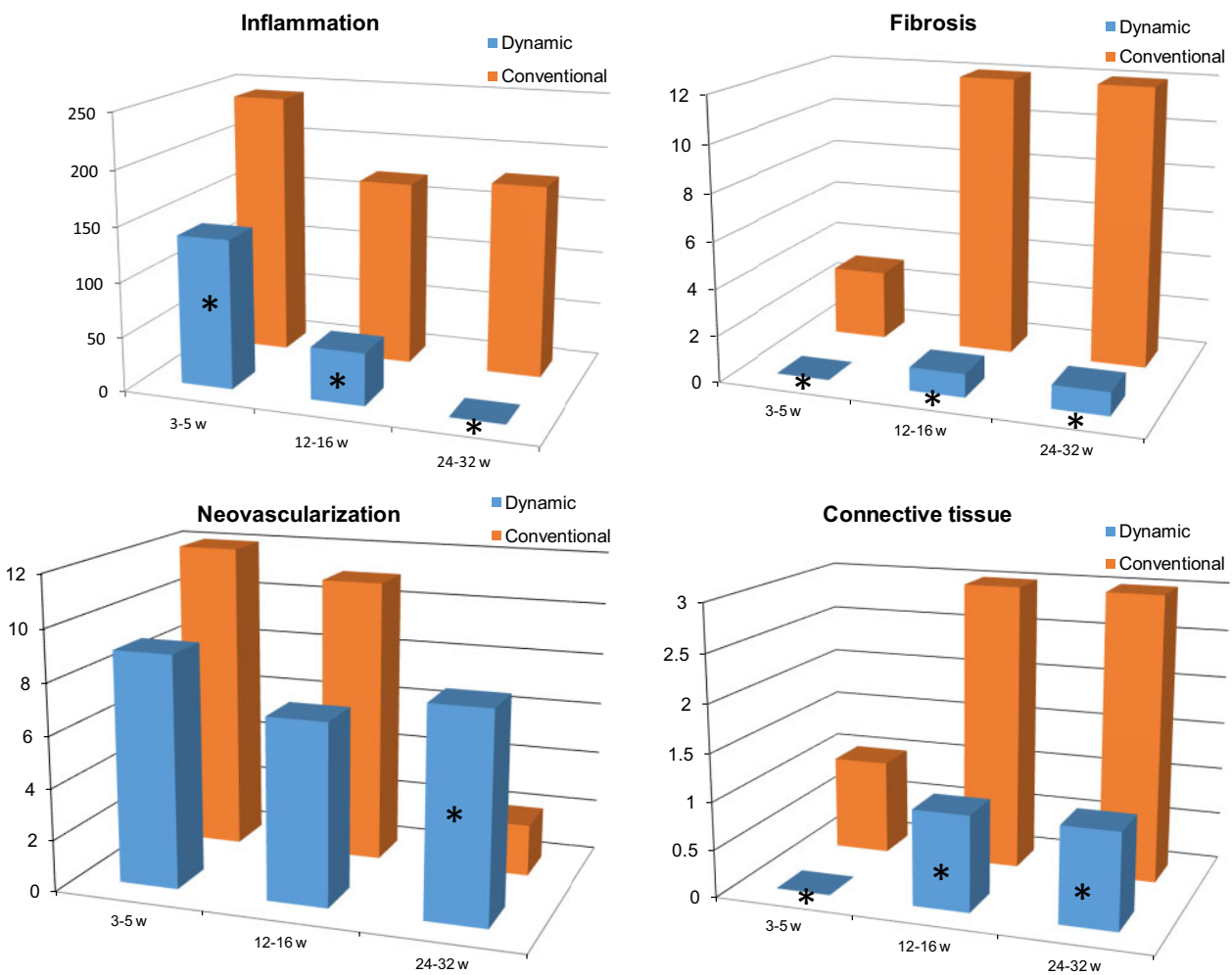

FIG. 2. Quantitative histopathological analysis: median values of parameters counted at the mesh-tissue interface are showed (for each group according to post-operative time); superscript * shows significant differences $(p<0.05)$.

quality and structure of the ingrown elements: viable and well-hydrated connective tissue on one end of the spectrum and stiff, hardened, disordered, and/or dehydrated fibrotic scarring on the other. Fibrosis and dense or loose collagen fibers were examined to determine the differences between hydrated connective tissue and scarred tissue; then, a specific quantitative assessment was carried out to determine the incorporation of fibrotic scar tissue. The development of these regressive elements was evaluated as absent (0-1 layers), mild (1-3 layers), moderate (4-10 layers), or abundant ( $>11$ layers).

\section{Elastic fibers and nervous structures}

No comparison was possible for the ingrowth of elastic elements within the implant structure, as these components were completely missing in the conventional mesh biopsies (as highlighted in the Results section). Similarly, as nervous elements were not detected in conventional implants, no

TABLE 1. Scoring criteria for histological analysis

\begin{tabular}{|c|c|c|c|c|}
\hline Score & 0 & 1 & 2 & 3 \\
\hline $\begin{array}{l}\text { Inflammatory } \\
\text { infiltration }\end{array}$ & $\begin{array}{l}\text { No cells in contact } \\
\text { with device }\end{array}$ & $\begin{array}{l}\text { Mild acute inflammation: } \\
\text { 30-80 lymphocytes and } \\
\text { neutrophils per HPF }\end{array}$ & $\begin{array}{l}\text { Severe acute inflammation: } \\
>150 \text { lymphocytes and } \\
\text { neutrophils per HPF }\end{array}$ & $\begin{array}{l}\text { Chronic inflammation: } \\
>100 \text { lymphocytes and } \\
\text { plasma cells per HPF }\end{array}$ \\
\hline Neovascularization & $\begin{array}{l}\text { No more than one blood } \\
\text { vessel per HPF }\end{array}$ & 2-4 blood vessels per HPF & 5-8 blood vessels per HPF & $\geq 9$ blood vessels per HPF \\
\hline $\begin{array}{l}\text { Connective tissue } \\
\text { ingrowth }\end{array}$ & $\begin{array}{l}\text { Well-hydrated, loose } \\
\text { connective tissue }\end{array}$ & $\begin{array}{l}\text { Well hydrated, loose } \\
\text { connective tissue. Mild } \\
\text { fibrosis }\end{array}$ & $\begin{array}{l}\text { Incorporation of dense, } \\
\text { compact, and disordered } \\
\text { connective tissue; } \\
\text { decrease in loose } \\
\text { connective tissue. }\end{array}$ & $\begin{array}{l}\text { Trabeculae of fibrous, } \\
\text { rigid, irregular, and } \\
\text { disorganized tissue; } \\
\text { loose connective tissue } \\
\text { absent }\end{array}$ \\
\hline Fibrosis & Absent & Mild: 1-3 layers thick & $\begin{array}{l}\text { Moderate: 4-10 layers } \\
\text { thick }\end{array}$ & Severe: >11 layers) \\
\hline
\end{tabular}

HPF, high power fields. 
TABLE 2. Histologic comparison between the inflammatory infiltrate of static and $3 D$ dynamic implants over time

\begin{tabular}{lcc}
\hline & \multicolumn{2}{c}{ Inflammatory response } \\
\hline Postoperative time & \multicolumn{1}{c}{ Conventional implant } & 3D dynamic implant \\
\hline 3-5 weeks & $\begin{array}{c}\text { Massive, homogeneous, of acute type (lymphocytes- } \\
\text { granulocytes); macrophages often detected }\end{array}$ & $\begin{array}{c}\text { Mild, of acute type (lymphocytes-granulocytes); } \\
\text { macrophages sometimes detected } \\
\text { 3-4 months }\end{array}$ \\
$\begin{array}{c}\text { Persistent, massive, of subacute type (lymphocytes-plasma } \\
\text { cells) } \\
\text { Persistent, moderate/elevated, of chronic type (plasma } \\
\text { cells-histiocytes) }\end{array}$ & $\begin{array}{c}\text { Absenticant; occasional elements of } \\
\text { subacute inflammation detected } \\
\text { Absent }\end{array}$ \\
\hline
\end{tabular}

comparative assessment of these structures was carried out.

\section{Statistics}

For each of the four variables (cellular infiltration, neovascularization, connective tissue, and fibrosis), 10 HPFs were scored according to the Valentin scoring system (13) as shown in Table 1, with less favorable outcomes represented as higher scores, except for neovascularization. Significant effects $(P<0.05)$ were determined using a nonparametric test, the Mann-Whitney $U$-test. SAS software (version 9.3) was used for all analyses, and results are expressed as median and range. As mentioned in the description of the histological assessments, no statistical comparison was possible for the elastic fibers or nervous ingrowth since these elements were consistently not detected in the conventional implants.

\section{RESULTS}

The differences between the two types of implants examined at various postoperative stages were noteworthy. The results regarding the specific tissue components are described below and schematically highlighted in Tables 2-6.

\section{Inflammatory infiltrate}

\section{Short term (3-5 weeks)}

In conventional, static flat meshes, histological examination showed that after 1 month, there was a large inflammatory reaction characterized by lymphocytes and granulocytes massively and homogeneously infiltrating the prosthetic structure (Fig. 3A). Conversely, the inflammatory reaction within the 3D dynamic implant at the same postoperative period was composed of the same cellular elements, but with only mild infiltration (Fig. 3B).

\section{Mid-term (3-4 months)}

In conventional, static prostheses, the inflammatory reaction was clearly persistent, having practically the same intensity here as for the short term. In this type of prosthetic, inflammation was subacute, mainly characterized by lymphocytes and plasma cells (Fig. 4A). In the same period, the inflammatory response within the 3D dynamic implant was virtually absent or irrelevant (Fig. 4B).

\section{Long term (6-8 months)}

The static meshes at this stage still presented with a persistent high-level inflammatory response, although of a lesser degree than at the mid-term. At this time,

TABLE 3. Histologic comparison of vascular ingrowth between static and $3 D$ dynamic implants over time

\begin{tabular}{|c|c|c|}
\hline \multicolumn{3}{|c|}{ Vascular structures } \\
\hline Postoperative time & Conventional implant & 3D dynamic implant \\
\hline $3-5$ weeks & $\begin{array}{l}\text { Presence of a large number of undifferentiated } \\
\text { neoangiogenic clusters }\end{array}$ & $\begin{array}{l}\text { Presence of a large number of neoangiogenic clusters, } \\
\text { having initial characterization in venous and arterial } \\
\text { structures }\end{array}$ \\
\hline 3-4 months & $\begin{array}{l}\text { Presence of a sufficient number of immature venous } \\
\text { and arterial structures }\end{array}$ & $\begin{array}{l}\text { Noteworthy increase in number and dimension of the } \\
\text { vascular elements showing a complete configuration } \\
\text { in all structural components, well-differentiated in } \\
\text { mature veins and arteries }\end{array}$ \\
\hline $6-8$ months & $\begin{array}{l}\text { Significant decrease of the vascular structures within } \\
\text { the flat mesh; the vascular elements, of capillary } \\
\text { type, are clearly there to support the reactive } \\
\text { inflammation still present within the implant tissue }\end{array}$ & $\begin{array}{l}\text { Abundant presence of mature and well-differentiated } \\
\text { vascular structures, often of large dimensions; } \\
\text { remarkable thickening of the arterial media, } \\
\text { compatible with a condition of compensatory } \\
\text { hyperplasia }\end{array}$ \\
\hline
\end{tabular}


TABLE 4. Histological comparison of connective tissue incorporation between static and 3D dynamic implants over time

\begin{tabular}{|c|c|c|}
\hline \multicolumn{3}{|c|}{ Connective tissue incorporation } \\
\hline Postoperative time & Conventional implant & 3D dynamic implant \\
\hline $3-5$ weeks & $\begin{array}{l}\text { Initial incorporation of amorphous connective tissue } \\
\text { with high water content }\end{array}$ & $\begin{array}{l}\text { Incorporation of lax connective tissue with high water } \\
\text { content }\end{array}$ \\
\hline 3-4 months & $\begin{array}{l}\text { Connective incorporation compact and almost } \\
\text { disordered, decreased vascular and water contents }\end{array}$ & $\begin{array}{l}\text { Further increase of loose and well-hydrated } \\
\text { connective tissue, organized in concentric or spiral } \\
\text { fashion; vascular structures markedly increased. }\end{array}$ \\
\hline $6-8$ months & $\begin{array}{l}\text { Ingrowth of irregular and disorganized stiff trabeculae } \\
\text { forming fibrotic plaques; water content decreased; } \\
\text { vascular structures sharply reduced; detected vessels } \\
\text { merely act as support for the reactive inflammatory } \\
\text { response }\end{array}$ & $\begin{array}{l}\text { Loose and well-hydrated connective tissue, organized } \\
\text { in concentric or spiral fashion; this structural } \\
\text { configuration resembles the response to the } \\
\text { dynamic impact exerted upon the device; } \\
\text { noteworthy amount of mature vascular structures }\end{array}$ \\
\hline
\end{tabular}

the inflammatory response clearly consisted of chronic elements, mostly lymphocytes, plasma cells, and histiocytes, with some multinucleated cells (Fig. 5A). The 3D dynamic implants, in contrast, lacked inflammatory infiltration, as no cellular elements of inflammation were detectable (Fig. 5B).

\section{Vascular structures}

\section{Short term}

In the early phase after implantation within the static meshes, many undifferentiated neoangiogenic spots could be detected (Fig. 6A). In the 3D dynamic implants, a consistent amount of neoangiogenic clusters appeared, showing an initial characterization of venous and arterial structures (Fig. 3B).

\section{Mid-term}

At this stage, the conventional prostheses showed the presence of a discrete number of vascular ele- ments, although they were not well differentiated as venous or arterial structures (Fig. 4A). The 3D dynamic implants showed a significant increase in the number and size of well-differentiated vascular structures, including veins and arteries. The ingrown vessels were functional in arterial perfusion and venous drainage (Figs. 6B and 9A).

\section{Long term}

After 6-8 months, a considerable reduction in the incorporation of vascular components in the static prostheses was noted. These elements were characterized as immature, capillary-type structures that were functionally linked to support the reactive inflammation that was still evident in the prosthetic structure (Figs. 7A and 9A). In contrast, during the same period, a large number of well-differentiated, mature vascular structures were found within the 3D dynamic implants, including all vascular constituents (intima, media, and adventitia),

TABLE 5. Histologic comparison of elastic fiber ingrowth between static and $3 D$ dynamic implant over time

\begin{tabular}{lcl}
\hline & & Elastic fibers \\
\hline Postoperatvive stage & Conventional implant & \\
\hline $3-5$ weeks & Missing & Ingrowth of numerous clusters of elastic fibers \\
$3-4$ months & Missing & Noteworthy increase in the amount of elastic fibers \\
$6-8$ months & Missing & Several nuclei of elastic fibers interspersed in whole prosthetic structure \\
\hline
\end{tabular}

TABLE 6. Histologic comparison of nervous structural ingrowth between static and $3 D$ dynamic implants over time

\begin{tabular}{lcc}
\hline Postoperative time & Conventional implant & Nervous structures \\
\hline $3-5$ weeks & Missing & $\begin{array}{c}\text { Numerous neurogenic spots with nervous elements in advanced phase of maturation; } \\
\text { spotty evidence of well-constituted nervous ganglia } \\
\text { Increased neurogenic incorporation amid the prosthetic structure; the newly formed } \\
\text { nervous elements were well-differentiated in all specific components (endoneurium, } \\
\text { perineurium) } \\
\text { Presence of numerous structurally mature nervous elements, provided by perineurium } \\
\text { and endoneurium, showing well-organized nervous fibers }\end{array}$ \\
\hline 6-8 months & Missing & 3D dynamic implant \\
\hline
\end{tabular}




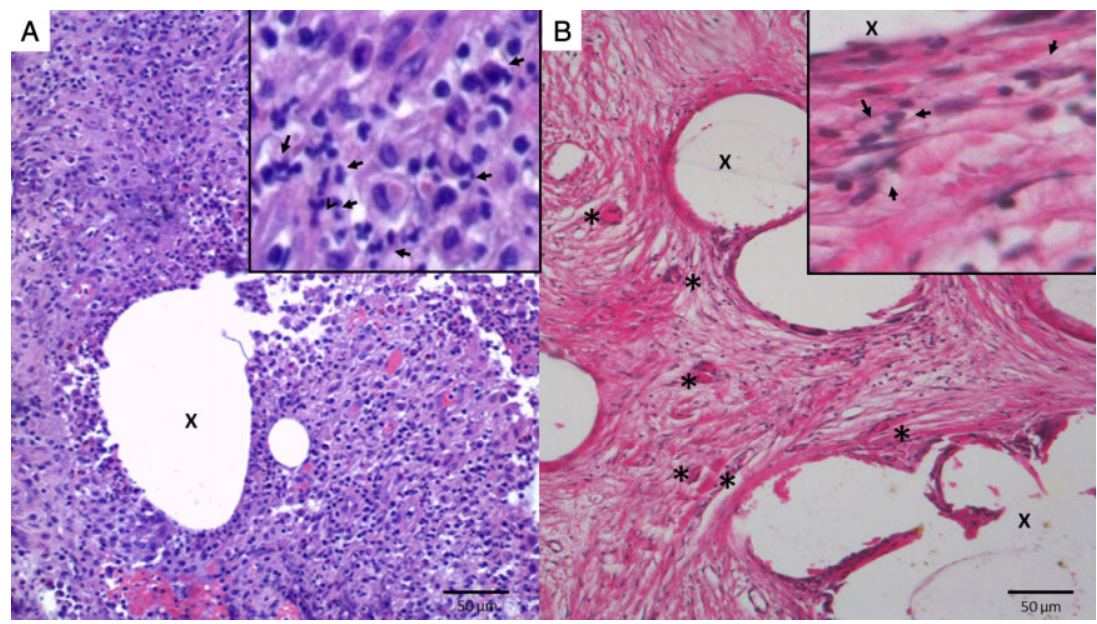

FIG. 3. Inflammatory response short term. Implant fibers marked with X. (A) Flat mesh excised 5 weeks post implantation. Intense inflammatory reaction of acute type, mostly composed by neutrophils (arrow inset). $\mathrm{EE} \times 20$. (B) Dynamic implant removed 4 weeks postop. Modest inflammatory infiltrate prevalently composed by lymphocytes (arrow inset). Numerous neoangiogenetic clusters are detectable in a fibrocollagenous surround $\left({ }^{*}\right)$. EE $\times 20$.

indicating a high rate of blood flow. The significant thickness of the muscular wall of the arteries was noteworthy, indicating a compensatory hyperplasia. This structural arrangement was probably develop- ing as a hemodynamic support to overcome the compression exerted ab extrinsico on the arteries during inguinal structure movement (Figs. 7B, 8B, 9B, and 9C).
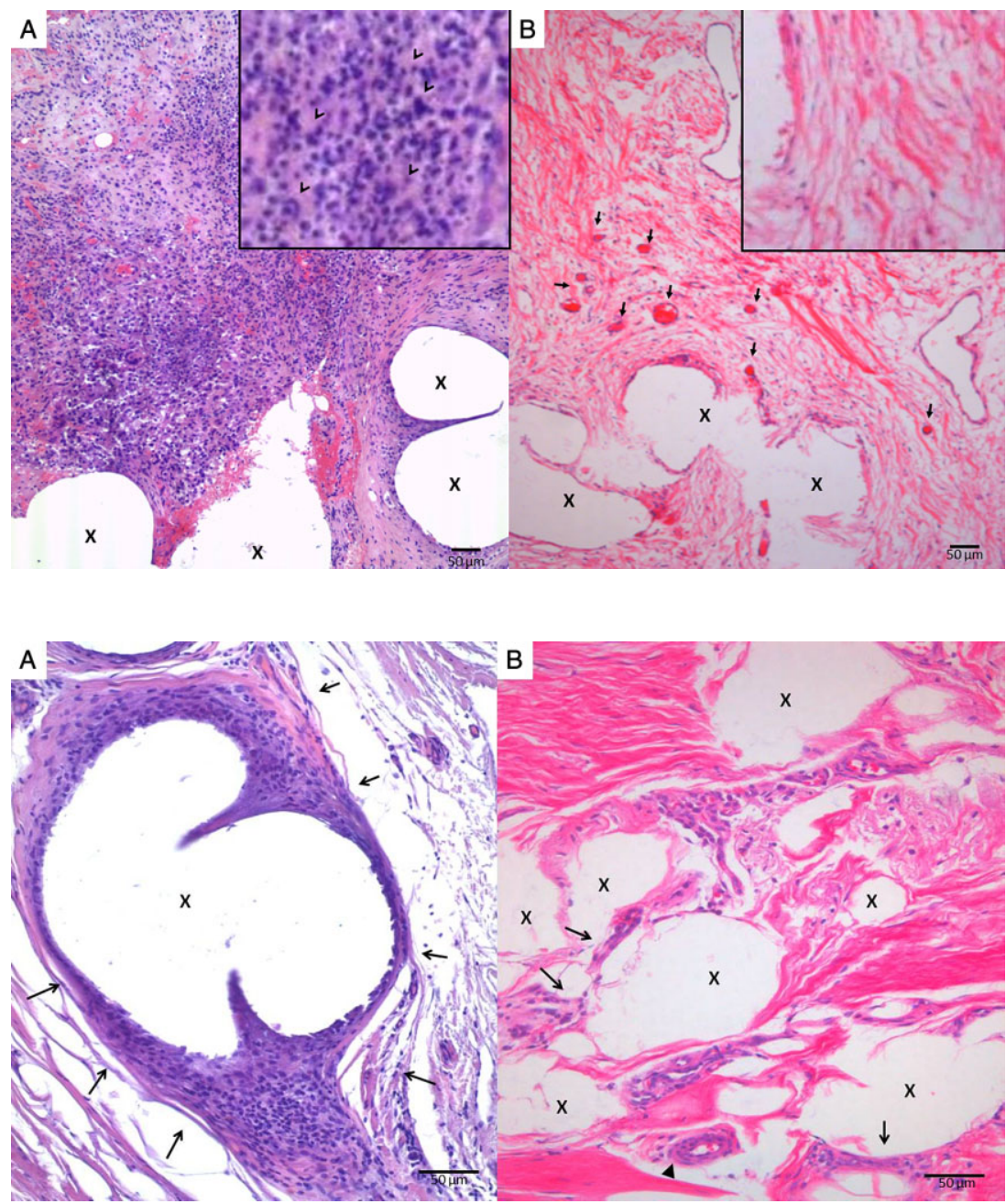

FIG. 4. Inflammatory response mid term. Implant fibers marked with $X$. (A) Flat mesh excised 3 months postop. Intense inflammatory infiltrate mostly composed by lymphocytes (arrowhead inset). Irrelevant neoangiogenesis Modest quote of connective ingrowth. $E E \times 10$. (B) Dynamic implant specimen excised 3 months postop. Lax and well hydrated connective ingrowth with intense vascularization (arrows). No inflammatory infiltrate detectable (inset). $\mathrm{EE} \times 10$.
FIG. 5. Inflammatory response long term. Implant fibers marked with X. (A) Flat mesh excised 8 months postop. Chronic inflammatory infiltrate mostly composed by lymphocytes, all around the mesh (arrow). Thin fibrotic strip with rare vascular structures formed by capillary vessels. EE 20x. (B) Specimen of dynamic implant excised 8 months after implantation. Lax and wellhydrated fibroconnective structure showing well differentiated vascular structures (arrowhead). Insignificant inflammatory infiltrate (arrow). EE 20x. 


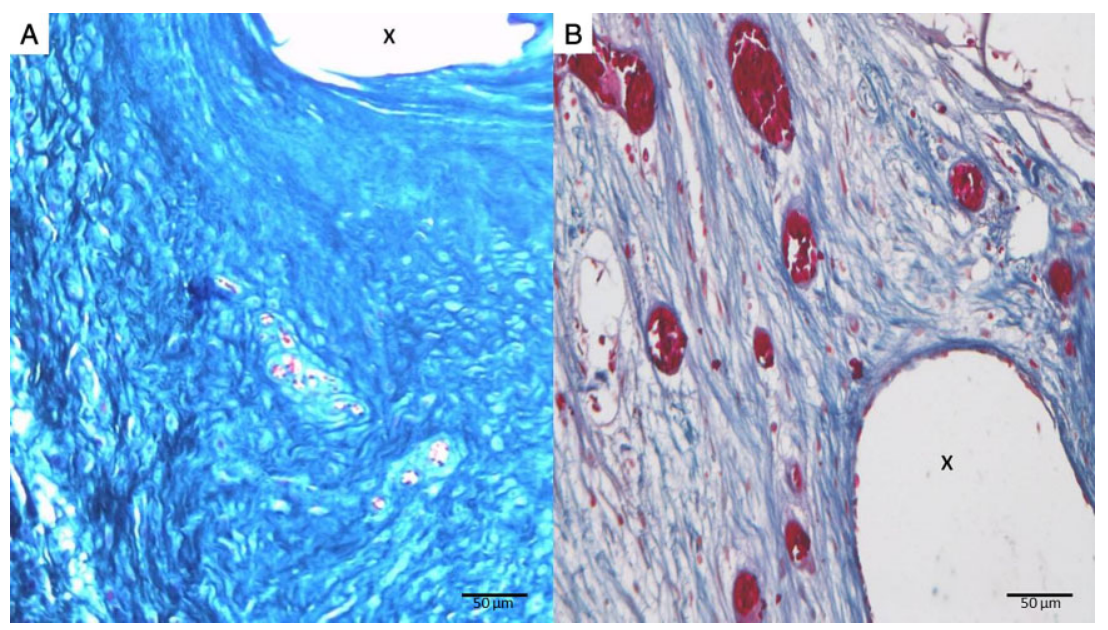

FIG. 6. Connective ingrowth short term. Implant fibers marked with X. (A) Flat mesh removed 6 weeks postoperatively. Marked connective ingrowth with almost disordered fiber arrangement and satisfactorily hydration. Limited neoangiogenesis. AM 20x. (B) Biopsy specimen of dynamic implant excised 4 weeks after implantation. Lax and well hydrated connective ingrowth with noteworthy amount of neoangiogenetic clusters. Absent inflammatory infiltrate. AM 20x.

\section{Connective tissue ingrowth}

\section{Short term}

After 1 month, the ingrowth of amorphous and well-hydrated connective tissue elements in the static prostheses was detectable. These fibers were arranged in a disorganized fashion, and the vascular support was limited (Fig. 6A). At the same time stage within the 3D dynamic prostheses, the incorporation of connective tissue assumed the characteristics of a
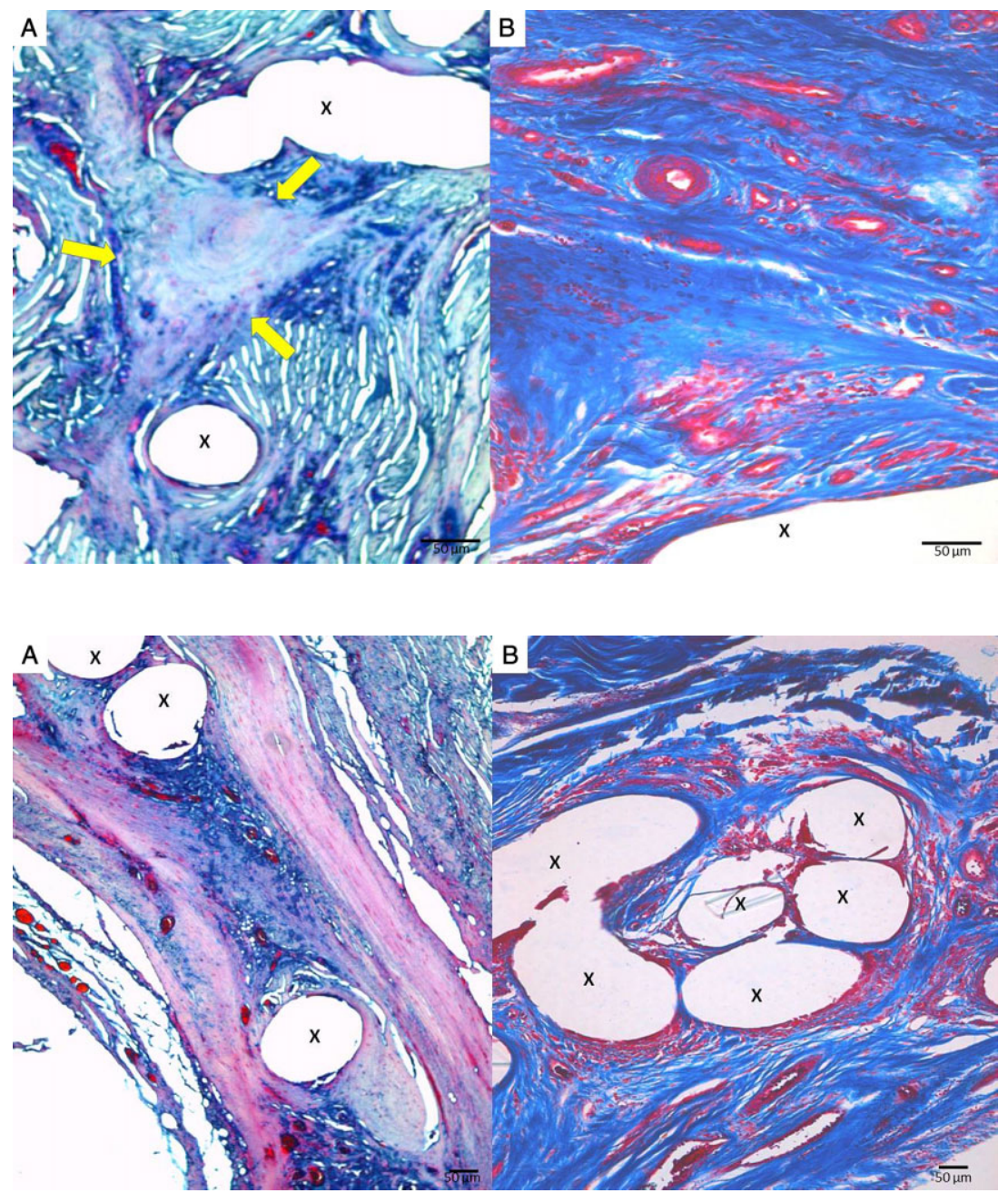

FIG. 7. Connective ingrowth long term. Implant fibers marked with X. (A) Flat mesh specimen removed 7 months after implantation. Dense fibrotic scar showing massive sclerohyalinosis. Limited vascular support exerted by small capillary vessels. AM 20x. (B) Dynamic implant. Biopsy specimen excised 6 months postop. Slack and well hydrated connective ingrowth showing a noteworthy amount of well-structured vascular components. AM 20x.
FIG. 8. Connective ingrowth long term. Implant fibers marked with X. (A) Flat mesh specimen removed 8 months after implantation. Marked dehydrated fibrotic plate with evident areas of sclerohyaline evolution. Inflammatory infiltrate supported by small capillary vessels. AM 10×. (B) Dynamic implant. Biopsy specimen excised 8 months postop. Lax and woven connective structures arranged in spiral fashion. Rich vascularization composed by numerous mature vascular elements. Insignificant inflammatory infiltrate. AM 10x. 


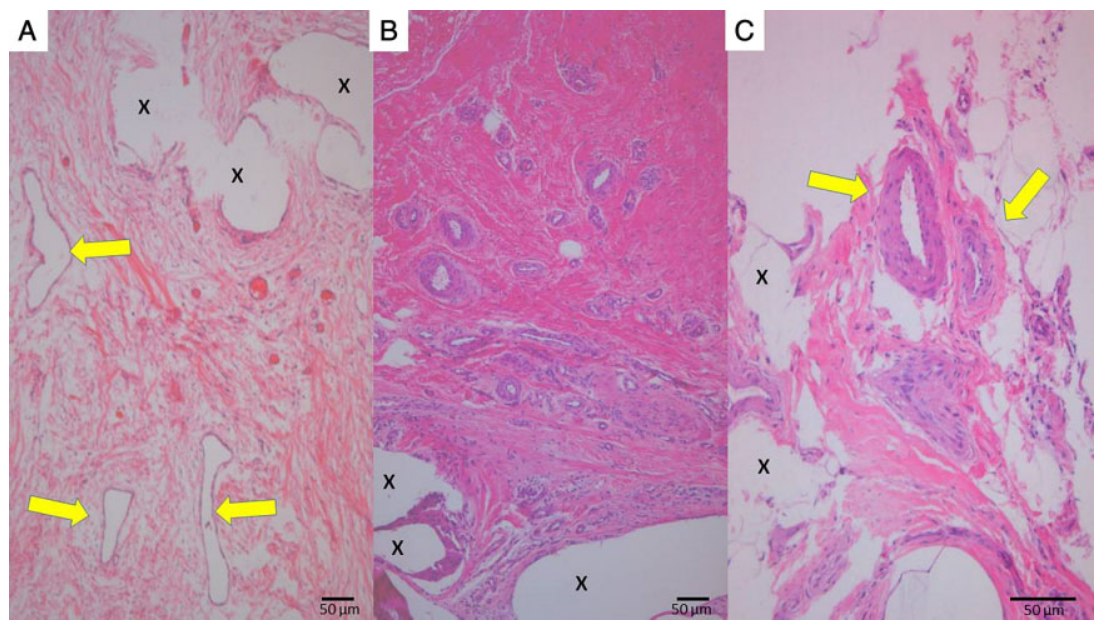

FIG. 9. Tissue incorporation in dynamic implants. Implant fibers marked with $\mathrm{X}$. (A) Biopsy specimen excised 4 months postoperative. Slack, well-hydrated and vascularized connective stroma showing broad vascular structures (arrows). EE 10x. (B) 7 months post implantation. Wellestablished and lax fibroconnective ingrowth with great amount of mature vascular structures. Many of the arteries show an uniform medial hyperplasia. Irrelevant inflammatory infiltrate among the implant fibers. EE 10x. (C) 8 months after implantation. Couple of large arteriolar structures (arrows) well constituted in all its components. EE 20x.

flourishing fibrocollagenous structure that was vascularized and well hydrated. These elements were arranged in regular contours (Fig. 6B).

\section{Mid-term}

The connective tissue incorporation in static implants at this stage appeared dense, compact, and somewhat messy, with sparse vascular elements. Compared with the initial phase, the hydration looked reduced. At the same time period, the 3D dynamic implant was infiltrated by connective tissue elements showing a loose, well-hydrated arrangement. The connective structures were excellently vascularized and organized into regular beams, sometimes in a spiral fashion.

\section{Long term}

The static mesh implants showed plaques of rigid fibrous material, having an irregular and disordered aspect. Extreme dehydration and limited vascularization were also evident. Among these fibrotic plaques, capillary vascular structures gave support to the reactive inflammatory response (Figs. 7A and 8A). In contrast, the 3D dynamic implants showed the presence of loose connective tissue, well hydrated and organized in concentric or spiral-shaped structures. This structural design seems likely due to the effect of dynamic compliance during movements. Numerous well-formed vascular structures were evident among the connective tissue (Figs. 7B and 8B).

\section{Elastic fibers}

\section{Short term}

There was an absence of elastic elements in the incorporated tissue in conventional prostheses. In the 3D dynamic responsive implants, clusters of elastic fibers disseminated in the proliferated tissue were clearly detected (Fig. 10A).

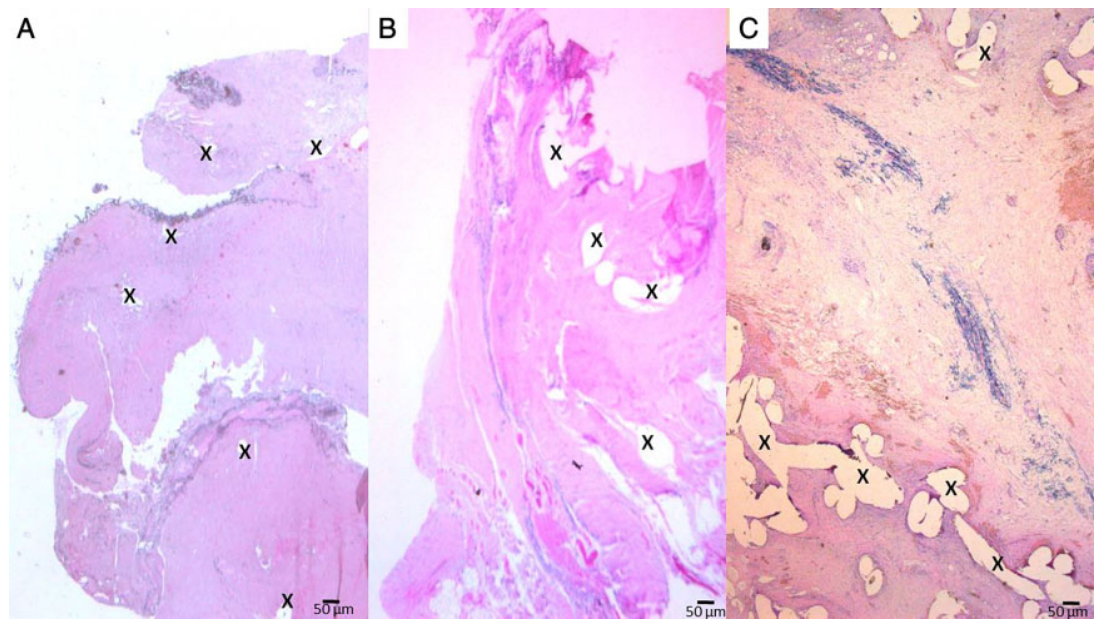

FIG. 10. Elastic fiber incorporation in dynamic implants. Implant fibers marked with $\mathrm{X}$, elastic elements colored in black. (A) Short term (5 weeks postop). Low magnification image showing the colonization of dense bundles of elastic elements at the edge of the biopsy specimen. WvG 2,5x. (B) Mid term (3 months postop) Increase of elastic nuclei arranged in multifocal elements. WvG 2,5×. (C) Long term (7 months postop.) Noteworthy proliferation of elastic structures organized in elongated bundles. WvG 2,5×. 


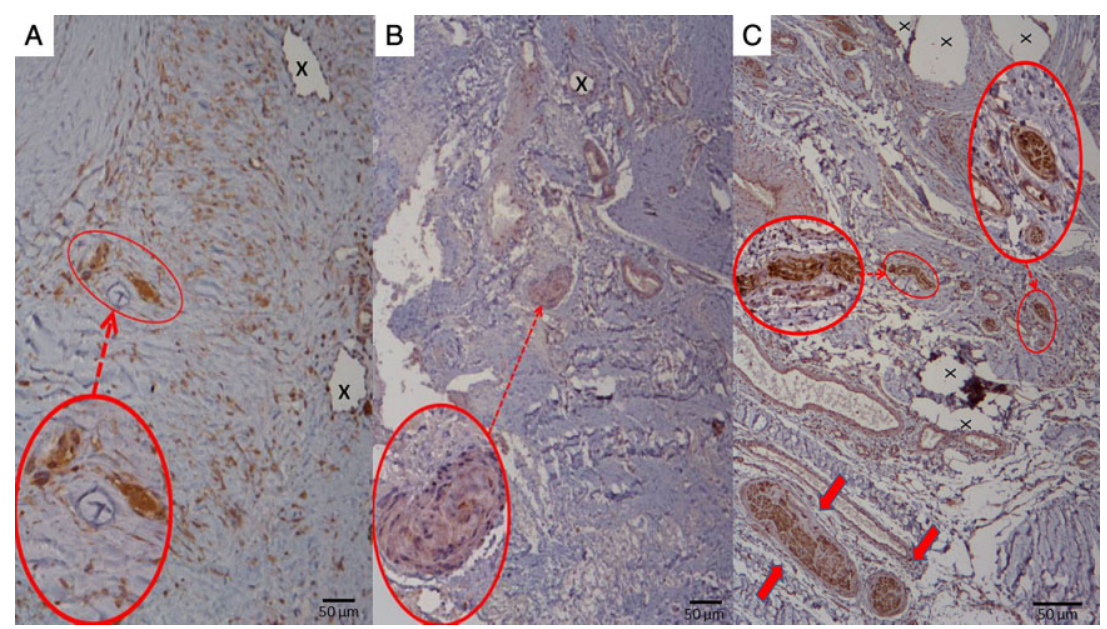

FIG. 11. Nervous structures ingrowth in dynamic implants. Implant fibers marked with X. (A) 1 month post implantation. Presence of several neurogenetic clusters (dark spots). 2 nervous elements (magnified $40 x$ in the circles) in evident structural evolution are found among the implant fibers. NSE 10x. (B) 3 months after implantation. Nervous structure in phase of intermediate maturation (magnified $40 x$ in the circles). NSE 10x. (C) 6 months postop. Well formed nervous structures (arrows and magnified $40 \times$ in the circles) mature in all its components seen in transverse and in sagittal section. NSE 20x.

\section{Mid-term}

At this stage, elastic elements were still missing in conventional prostheses. In the newly formed tissue of the 3D dynamic implants, a noticeable increase in the amount of elastic fibers was seen (Fig. 10B).

\section{Long term}

Elastic elements were not found in the static prosthesis. In the same time period, the tissue incorporated within the 3D dynamic implant clearly contained numerous, interspersed spots of elastic fibers all around the implant (Fig. 10C).

\section{Nervous structures}

\section{Short term}

Neural elements were absent in the incorporated tissue of the static implants. In this early phase, the 3D dynamic implants showed numerous neurogenic clusters with the characteristics of neural structures in progressive stages of maturity (Figs. 11A and 12A). Occasionally, well-defined gangliar structures could be identified (Fig. 12B).

\section{Mid-term}

At this stage, no nervous structures were found in the newly formed tissue of the static meshes. In the $3 \mathrm{D}$ dynamic implants, there was a marked increase in neurogenesis. The neural structures were at an advanced stage of maturation and differentiation of all the specific components (endo- and perineurium) (Fig. 11B).

\section{Long term}

No neural elements were found in conventional static implants, even at the long term. At the same postoperative time period, the newly formed tissue within the dynamic prostheses stood out as containing a large amount of structurally complete nerve

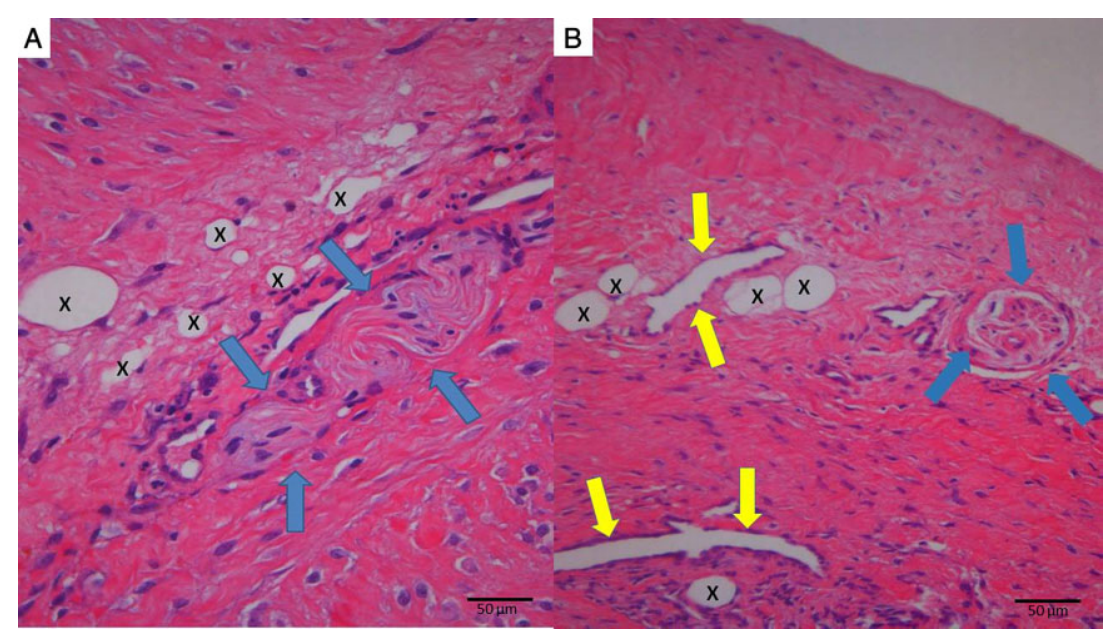

FIG. 12. Tissue incorporation in dynamic implants. Implant fibers marked with $X$. (A) Biopsy excised 4 weeks post implantation. Nervous axon in development phase (arrows) in the context of well vascularized connective proliferation among the implant fibers. EE 20×. (B) Biopsy excised 5 weeks post implantation. Nervous ganglion (arrow) and broad venous structures (arrows) in the contest of connective proliferation among the implant fibers. EE 20x. 
TABLE 7. Median scores and range for each group at the different postoperative times

\begin{tabular}{|c|c|c|c|c|}
\hline \multirow[b]{2}{*}{ Criteria } & & \multicolumn{3}{|c|}{ Postoperative stage } \\
\hline & & Short term & Mid-term & Long term \\
\hline \multirow[t]{2}{*}{ Inflammatory infiltration } & Conventional flat mesh & $3(3-2)^{*}$ & $2(2-1)^{*}$ & $3(3-2)^{*}$ \\
\hline & 3D dynamic implant & $1(2-1)^{*}$ & $1(1) *$ & $0(1-0) *$ \\
\hline \multirow[t]{2}{*}{ Fibrosis } & Conventional flat mesh & $1(2-1)^{*}$ & $3(3-2) *$ & $3(3-2) *$ \\
\hline & 3D dynamic implant & $0(1-0)^{*}$ & $1(1-0)^{*}$ & $0(1-0)^{*}$ \\
\hline \multirow[t]{2}{*}{ Neovascularization } & Conventional flat mesh & $3(3-2)$ & $3(3-2)$ & $1(1-1)^{*}$ \\
\hline & 3D dynamic implant & $3(3-2)$ & $2(3-1)$ & $2(2)^{*}$ \\
\hline \multirow[t]{2}{*}{ Connective tissue } & Conventional flat mesh & $2(2-1) *$ & $2(3-1) *$ & $3(3-2) *$ \\
\hline & 3D dynamic implant & $0(1-0)^{*}$ & $1(1-0)^{*}$ & $0(1-0) *$ \\
\hline
\end{tabular}

$* P<0.05$.

elements, consisting of well-structured axons having regular conforming peri- and endoneurium (Fig. 11C).

\section{Statistical results}

Median scores of inflammatory infiltrate, fibrosis, neovascularization, and connective tissue on all biopsies at different times (short, mid-, and long terms), were compared with the Mann-Whitney $U$-test. The median scores for each group by postoperative time are shown in Table 7, and the quantitative histological results are shown in Fig. 2. Hypothesis testing showed statistically significant differences between conventional flat meshes and 3D dynamic implants $(P<0.05)$. Analysis of median values confirmed that inflammatory infiltrate and fibrosis in the dynamic implant was lower than in the conventional one, although with a higher range and, consequently, a greater variability.

The most intense response, as judged by the number of cells at the implant site over all time intervals, was observed in static implants. Vascularization showed a significant difference only during the longterm period (6-8 months).

\section{DISCUSSION}

Significant developments have been made in recent decades in prosthetic inguinal hernia repair. The use of biologically compatible implants, aimed at reinforcing the inguinal region weakened by hernia protrusion, has greatly improved the results of surgical treatment of this disease. Although there are undisputed benefits, including the reduction of recurrence, the scientific literature in recent years has shown a high rate of complications and other adverse events deriving from the use of conventional prostheses. Among the typical complications of hernia repair, such as tissue tear, bleeding, hematoma, and infection (mostly due to fixation), specific meshrelated side effects have recently been highlighted as a source of concern among surgeons. The growing number of studies reporting long-acting postoperative discomfort and chronic pain confirm these concerns $(4,6,14)$. To date, the scientific community and industry have not been able to provide answers or solutions to resolve these issues. Perhaps the problem lies in the static characteristics of conventional prostheses. Flat meshes and plugs are all static devices, and being passive and dynamically inert, do not take part in the natural movements of the inguinal region. Therefore, these implants are not integrated with the dynamic nature of the inguinal region. Indeed, the myotendinous structure of the groin is considered one of the most mobile parts of the body, being subjected to tractions arising from the lower limb and the abdominal wall, as well as to visceral pulsing due to pressure changes in the peritoneal cavity. Consequently, it may be reasonable to assume that conventional prosthetics are physiodynamically inadequate to repair the affected groin. Another important issue in modern prosthetic hernia repair is the poor quality of tissue ingrowth within conventional implants. The biologic response to conventional implants has been described in a large number of scientific articles (15-22). These studies clearly highlight the poor quality of tissue incorporation in conventional prostheses utilized for inguinal hernia repair. The term "scar plate" used by many authors well indicates the stiff, fibrotic scar formation that is the typical product of these devices. The ingrowth of this kind of regressive scar tissue leads to the well-known phenomenon of mesh shrinking. The reduction of the mesh surface area due to implant shrinkage (up to $30 \%$ ) has been identified as a significant factor in recurrence $(19,23)$.

In recent years, a newly designed 3D polypropylene implant was developed and used to repair inguinal hernias (23). This device is inherently dynamic and self-repairing, which should theoretically eliminate many complications linked with invasive fixation. In addition, evidence from animal studies has 
shown that the dynamic responsive structure of this $3 \mathrm{D}$ prosthesis induces excellent quality of tissue ingrowth, very different from the shrunken and stiff fibrotic scar plates of the conventional meshes (12).

The aim of this work was to highlight the difference in biologic response between the conventional static implants and dynamic responsive prostheses. The study was conceived in order to assess tissue incorporation in both types of devices at short, midand long terms after implantation. The long-term limit of 6-8 months has been defined in the literature as the time frame ( $\sim 6$ months) in which the tissue incorporation of conventional flat meshes ends and all tissue changes appear definitely established $(15,16,22)$.

The results from the histological comparison between the static and the conventional implants show that the implant structure is important in defining the architecture of the biologic response. By analyzing the individual elements of scar formation, it becomes possible to assess the differences in biologic response between the two categories of prosthesis.

In terms of inflammatory response, a noteworthy inflammatory infiltration of acute type in the early stage (ca. 1 month postoperative), constituted by lymphocytes and granulocytes, was clearly evident in conventional meshes; within the 3D dynamic implants, however, the inflammatory response was greatly reduced. Macrophages were detected more often within the static meshes than in the 3D dynamic implant. In the mid- and long terms, the inflammatory elements in conventional implants slightly decreased. Nevertheless, the typical components of chronic inflammation, such as histiocytes and plasma cells, were still clearly evident several months after implantation within conventional static prostheses. As the flat meshes appear not to be physiologically integrated among the groin structures, the inflammatory infiltrate consequently displays typical features of the chronic foreign body reaction. The infiltration persists for a long time because the implant never integrates with the groin during movement. When chronic inflammation is present in a body area, discomfort and pain are often clinically symptomatic. These features clearly contrast with the lack of evidence for inflammatory infiltrate in the 3D dynamic implants at both 2-3 months postoperative and at the long term. This effect seems to be a consequence of the dynamic compliance of the 3D implant, which appears to allow a complete physiological integration during movements.

Significant differences were observed in connective tissue ingrowth by examining the histological results. After an almost identical progression of con- nective tissue incorporation at the short-term time point, the differences were clearly perceivable starting from the mid-term until 6-8 months postoperatively. In fact, at 2-3 months after implantation, the incorporated connective tissue of conventional meshes contained progressively less water, becoming irregular, disordered, stiff, and fibrotic. At this stage, scar formation is nearly accomplished, and the result is an inelastic fibrotic scar plate. Again, the mechanical properties of this fixed plaque contrast with the dynamic properties of the groin, which represents a physiological incongruence that could lead to discomfort during movements.

In contrast, the connective ingrowth within the 3D dynamic implant showed a constant, uninterrupted progression of viable connective elements. These were soft, loose, well-hydrated, and increasingly supported by mature vascular elements for the improved needs of blood perfusion. Even at the mid-term, the particular arrangement of the architecture of the newly formed connective fibers demonstrates organization in regular concentric elements or grouped in a spiral fashion. This well-ordered structural configuration suggests a response to the dynamic behavior of the device in compliance with the groin movements. This progressive incorporation of slack connective tissue within the $3 \mathrm{D}$ implant evidently inhibits the formation of the regressive fibrotic scar plate seen in the conventional meshes. This could also explain why, as highlighted in the experimental model, the dynamic prosthesis does not shrink (12).

Another interesting point from the histological analysis is the great discrepancy between the ingrowth vascular structures in both implants, particularly at the mid- and long terms. In fact, while in the short term a similar number of neoangiogenic clusters were seen in both implant types, from this point onward the differences became evident. After 3 months in conventional meshes, a progressive reduction of the vascular structures begins that continues well beyond the 6-month period. At this stage, the scar process is almost complete, and the stiff, fibrotic, and avascularized scar plate is the final product of the tissue incorporation. For this reason, there is no need for an abundant blood supply. The vascular structures at this phase were mostly capillaries, only supporting the persistent chronic inflammatory reaction within the implant fibers.

Conversely, angiogenesis within the 3D implants continued to progress. Vascularization began with undifferentiated vascular clusters detected in the early weeks after implantation. During the mid-term, these vascular structures differentiated into veins and arteries. Further evolution of the vascular elements 
was clearly observed in the long term. At this stage, newly formed veins and arteries displayed typical venous and arterial architecture. One particular feature was noted concerning the arterial structures, especially in the long-term specimens: a thickened muscular layer of the arterial structure was often seen that resembled the effects in arteries subjected to permanent cyclical loading. This effect seems to correspond to dynamic compliance with groin movements.

The dynamic compliance to the movements of the inguinal region could probably explain the progressive ingrowth of elastic fibers within the 3D dynamic implant. The development of elastic elements starting from the early stage was constant and gradual, but continuous, and increased with time until the long-term time point. No elastic structures were seen at any time in the conventional, static meshes. The progressive ingrowth of elastic elements among the 3D implant structures seems to confirm the benefits of the dynamically compliant behavior of the device.

Another element that crucially distinguishes the biologic response between the two types of implants is the ingrowth of nervous structures. Newly formed neural elements have never been detected in the incorporated tissues of the conventional flat meshes. On the contrary, the presence of neoneurogenic clusters within the $3 \mathrm{D}$ prostheses was apparent beginning from the first month. These structures progressively assume the typical composition of nervous elements, well-formed in all their components. Moreover, a thickened myelin sheath that resembles the typical modification of nervous structures subjected to cyclical compression was detected in long-term specimens. The thickening of the myelin sheath of the newly formed nerves, the medial hyperplasia detected in the muscular wall of the arteries, and the existence of a large amount of elastic fibers observed in the histology specimens of the 3D implants all indicate a long-acting regenerative response to compressive load. These hypotheses have been validated in the literature $(12,24-28)$.

\section{CONCLUSION}

The results of this comparative study contribute to our knowledge of the process of tissue incorporation. These outcomes show that an adequate implant design is important to induce good-quality tissue incorporation within the prostheses. From the results, it is clear that a prosthetic response to load, here a long-duration cyclical load in compliance with the surrounding tissue components, is essential to induce a well-formed tissue ingrowth, ensuring that new tissue development mimics the neighboring structures.

The histological progression of ingrown tissue much more closely resembles the natural abdominal wall components and structures in the $3 \mathrm{D}$ dynamic implants. All these characteristics are completely missing in the conventional static implants, where the biologic response leads to a persistent inflammatory reaction and produces thin, stiff, avascular, and dehydrated fibrotic scars, typical results of a regressive process.

Finally, we believe that because inguinal protrusions are degenerative processes, prosthetics used for hernia repair should address this pathogenesis of the disease by acting as regenerative scaffolds that promote new tissue ingrowth, not a regressive fibrotic scar plate.

Conflict of Interest: GA is the inventor of the 3D dynamic implant. The remaining authors have no conflicts of interest to report.

\section{REFERENCES}

1. Rutkow IM. Demographic and socioeconomic aspects of hernia repair in the United States in 2003. Surg Clin North Am 2003;83:1045-51.

2. Schwab R, Conze J, Willms A, Klinge U, Becker HP, Schumpelick V. Management of recurrent inguinal hernia after previous mesh repair: a challenge. Chirurg 2006;77:52330.

3. Amid PK. Causes, prevention, and surgical treatment of postherniorrhaphy neuropathic inguinodynia: triple neurectomy with proximal end implantation. Hernia 2004;8:343-9.

4. O'Dwyer PJ, Kingsnorth AN, Mohillo RG, Small PK, Lammers B, Horeysee G. Randomized clinical trial assessing impact of a lightweight or heavyweight mesh on chronic pain after inguinal hernia repair. Br J Surg 2005;92:166-70.

5. Rutkow IM, Robbins AW. Mesh plug hernia repair: a follow-up report. Surgery 1995;117:597-8.

6. Aasvang E, Kehlet H. Surgical management of chronic pain after inguinal hernia repair. Br J Surg 2005;92:795-801.

7. Amato G, Marasa L, Sciacchitano T, et al. Histological findings of the internal inguinal ring in patients having indirect inguinal hernia. Hernia 2009;13:259-62.

8. Amato G, Ober E, Romano G, et al. Nerve degeneration in inguinal hernia specimens. Hernia 2011;15:53-8.

9. Amato G, Romano G, Salamone G, et al. Damage to the vascular structures in inguinal hernia specimens. Hernia 2012; $16: 63-7$

10. Amato G, Agrusa A, Romano G, et al. Histological findings in direct inguinal hernia. Hernia 2013;17:757-63.

11. Amato G, Agrusa A, Romano G, et al. Muscle degeneration in inguinal hernia specimens. Hernia 2012;16:327-31.

12. Amato G, Lo Monte AI, Cassata G, Damiano G, Romano G, Bussani R. A new prosthetic implant for inguinal hernia repair: its features in a porcine experimental model. Artif Organs 2011;35:E181-90.

13. Valentin JE, Badylak JS, McCabe GP, Badylak SF. Extracellular matrix bioscaffolds for orthopaedic applications. A comparative histologic study. J Bone Joint Surg Am 2006;88: 2673-86.

14. Kim-Fuchs C, Angst E, Vorburger S, Heibling C, Candinas D, Schlumpf R. Prospective randomized trial comparing sutured 
with sutureless mesh fixation for Lichtenstein hernia repair: long-term results. Hernia 2012;16:21-7.

15. Read RC. Recent advances in the repair of groin herniation. Curr Probl Surg 2003;40:13-79.

16. Amid PK, Shulman AG, Lichtenstein IL, Hakakha M. Biomaterials for abdominal wall hernia surgery and principles of their applications. Langenbecks Arch Chir 1994;379:168-71.

17. EU Hernia Trialist Collaboration. Mesh compared with nonmesh methods on open groin hernia repair. Systematic review of randomized controlled trial. Br J Surg 2000;87:854-9.

18. Debord JR. The historical development of prosthetics in hernia surgery. Surg Clin North Am 1998;78:973-1006.

19. Klinge U, Klosterehalfen B, Muller M, Ottinger AP, Schumpelick V. Shrinking of polypropylene mesh in vivo: an experimental study in dogs. Eur J Surg 1998;164:965-9.

20. Amid PK. Lichtenstein tension-free hernioplasty: its inception, evolution, and principles. Hernia 2004;8:1-7.

21. Klosterhalfen $\mathrm{B}$, Junge $\mathrm{K}$, Klinge $\mathrm{U}$. The lightweight and large porous mesh concept for hernia repair. Expert Rev Med Devices 2005;2:103-17.
22. Klosterhalfen B, Klinge U, Schumpelick V. Functional and morphological evaluation of different polypropylene-mesh modifications for abdominal wall repair. Biomaterials 1998;19: 2235-46.

23. Amid PK. Shrinkage: fake or fact? In: Schumpelick V, Nyhus LM, eds. Meshes: Benefits and Risks. Berlin: Springer, 2004; 198-206.

24. Amato G, Agrusa A, Romano G. Fixation-free inguinal hernia repair using a dynamic self-retaining implant. Surg Technol Int 2012;22:107-12.

25. Tozzi P. The physiology of blood flow and artery wall. In: Sutureless Anastomoses. Darmstadt: Steinkopff, 2007;1224.

26. Kumar V, Abbas AK, Fausto N, eds. Robbins \& Cotran Pathologic Basis of Disease. Philadelphia: Saunders, 2005; 513-5.

27. Penkert G. Nerve compression syndrome-1. Chirurg 1998;69: 1114-22.

28. Kincaid JC, Stewart JD. Focal peripheral neuropathies. J Clin Neuromuscul Dis 1999;1:113. 